\title{
IRREDUCIBILITY OF EXTENSIONS OF LAGUERRE POLYNOMIALS
}

\author{
SHANTA LAISHRAM, SARANYA G. NAIR AND T. N. SHOREY
}

\begin{abstract}
For integers $a_{0}, a_{1}, \ldots, a_{n}$ with $\left|a_{0} a_{n}\right|=1$ and either $\alpha=u$ with $1 \leq u \leq 50$ or $\alpha=u+\frac{1}{2}$ with $1 \leq u \leq 45$, we prove that $\psi_{n}^{(\alpha)}\left(x ; a_{0}, a_{1}, \cdots, a_{n}\right)$ is irreducible except for an explicit finite set of pairs $(u, n)$. Furthermore all the exceptions other than $n=2^{12}, \alpha=89 / 2$ are necessary. The above result with $0 \leq \alpha \leq 10$ is due to Filaseta, Finch and Leidy and with $\alpha \in\{-1 / 2,1 / 2\}$ due to Schur.
\end{abstract}

\section{INTRODUCTION}

For positive integer $n$ and real number $\alpha$, the generalized Laguerre polynomial is given by

$$
L_{n}^{(\alpha)}(x)=\sum_{j=0}^{n} \frac{(n+\alpha)(n-1+\alpha) \ldots(j+1+\alpha)}{(n-j) ! j !}(-x)^{j}
$$

and $L_{n}^{(0)}(x)$ is called Laguerre polynomial. We shall restrict ourselves to the case when $\alpha$ is a rational number written uniquely as

$$
\alpha=\alpha(u)=u+\frac{a}{d}
$$

where $u, a, d \in \mathbb{Z}$ and $d \geq 1$ such that $a=0$ if $d=1$ and $1 \leq a<d, \operatorname{gcd}(a, d)=1$ if $d>1$. Thus $\alpha=u$ if $d=1$. The generalized Laguerre polynomial satisfies second order linear differential equation

$$
x y^{\prime \prime}+(\alpha+1-x) y^{\prime}+n y=0, y=L_{n}^{(\alpha)}(x)
$$

and the difference equation

$$
L_{n}^{(\alpha)}(x)-L_{n}^{(\alpha-1)}(x)=L_{n-1}^{(\alpha)}(x) .
$$

They have been studied in various branches of mathematics and mathematical physics and there is an extensive literature on them, see [16]. Schur [15], 14] was the first to establish interesting and important algebraic properties of these polynomials. In particular, the irreducibility of these polynomials has been well investigated, see [9] for an account of results in this connection. Filaseta, Finch and Leidy [4] showed that $L_{n}^{(\alpha)}(x)$ is irreducible for all $n$ and integers $\alpha$ with $0 \leq \alpha \leq 10$ except when $(n, \alpha) \in\{(2,2),(4,5),(2,7)\}$. Laishram and Shorey [8] extended it for integers $\alpha$

2000 Mathematics Subject Classification: Primary 11A41, 11B25, 11N05, 11N13, 11C08, $11 \mathrm{Z} 05$. Keywords: Irreducibility, Laguerre Polynomials, Primes, Newton Polygons. 
with $0 \leq \alpha \leq 50$ and showed that $L_{n}^{(\alpha)}(x)$ is irreducible for all $n$ except for $n=$ $2, \alpha \in\{2,7,14,23,34,47\}$ and $n=4, \alpha \in\{5,23\}$ where it has a linear factor. Further explicit factorizations for these exceptions have been given. In this paper, we consider more general polynomials than $L_{n}^{(\alpha)}(x)$. By irreducibility of a polynomial, we shall always mean that it is irreducible over $\mathbb{Q}$. Observe that if a polynomial of degree $m$ has a factor of degree $k$, then it has also a factor of degree $m-k$. Therefore given $a$ polynomial of degree $m$, we always consider a factor of degree $k$ where $1 \leq k \leq \frac{m}{2}$. Let $a_{0}, a_{1}, \ldots, a_{n}$ be integers with $\left|a_{0}\right|=\left|a_{n}\right|=1$ and $\alpha$ be a rational number given by (11). Then we define

$$
\begin{aligned}
\psi_{n}^{(\alpha)}(x)=\psi_{n}^{(\alpha)}\left(x ; a_{0}, a_{1}, \ldots, a_{n}\right) & =\sum_{j=0}^{n} a_{j}\left(\begin{array}{c}
n \\
j
\end{array}\right)(n+\alpha) \cdots(j+1+\alpha) d^{n-j} x^{j} \\
& =\sum_{j=0}^{n} a_{j}\left(\begin{array}{c}
n \\
j
\end{array}\right)(a+(u+n) d) \cdots(a+(u+j+1) d) x^{j}
\end{aligned}
$$

by (11). We observe that

$$
\psi_{n}^{(\alpha)}(x)=d^{n} n ! L_{n}^{(\alpha)}\left(\frac{x}{d}\right) \text { if } a_{j}=(-1)^{j}
$$

and therefore, $\psi_{n}^{(\alpha)}(x)$ with $a_{j}=(-1)^{j}$ is irreducible if and only if $L_{n}^{(\alpha)}(x)$ is irreducible. Thus the irreducibility of $\psi_{n}^{(\alpha)}(x)$ implies the irreducibility of $L_{n}^{(\alpha)}(x)$. Filaseta, Finch and Leidy [4] showed that $\psi_{n}^{(\alpha)}(x)$ with $0 \leq \alpha \leq 10$ and $\left|a_{0} a_{n}\right|=1$ is irreducible for all $n$ except $(n, \alpha) \in\{(2,2),(2,7),(4,4),(4,5),(8,8),(24,8)\}$ where $\psi_{n}^{(\alpha)}(x)$ has a linear factor. They proved that these exceptions are necessary in the sense that there exist integers $a_{0}, a_{1}, \ldots, a_{n}$ with $\left|a_{0}\right|=\left|a_{n}\right|=1$ such that $\psi_{n}^{(\alpha)}(x)$ has a linear factor. We extend their results for $\alpha \leq 50$. Let

$$
\begin{aligned}
\Omega= & (2,14),(2,23),(2,34),(2,47),(4,14),(4,20),(4,23),(6,44),(8,41),(12,24), \\
& (16,16),(16,20),(16,24),(16,29),(24,24),(30,24),(32,32),(32,48),(40,24), \\
& (48,24),(112,48),(120,24)\} .
\end{aligned}
$$

Theorem 1. Let $11 \leq \alpha \leq 50$ be an integer and $\left|a_{0} a_{n}\right|=1$. Then $\psi_{n}^{(\alpha)}(x)$ is irreducible except when $(n, \alpha) \in \Omega$ where it may have a linear factor or $(n, \alpha)=$ $(16,24)$ where it may have a quadratic factor. Further for every $(n, \alpha) \in \Omega$, there exist integers $a_{0}, a_{1}, \ldots, a_{n}$ with $\left|a_{0}\right|=\left|a_{n}\right|=1$ such that $\psi_{n}^{(\alpha)}(x)$ has a linear factor and further integers $a_{0}, a_{1}, \ldots, a_{n}$ with $\left|a_{0}\right|=\left|a_{n}\right|=1$ such that $\psi_{n}^{(\alpha)}(x)$ has a quadratic factor for $(n, \alpha)=(16,24)$. The factors for each case are given in the following table. 


\begin{tabular}{|c|c|}
\hline$x \pm b$ & $(n, \alpha)$ \\
\hline$x \pm 2$ & $(16,16),(32,32)$ \\
\hline$x \pm 4$ & $(2,14)$ \\
\hline$x \pm 6$ & $(2,34),(4,14),(4,20),(4,23)$, \\
& $(12,24),(16,20),(24,24),(48,24)$ \\
\hline$x \pm 10$ & $(32,48)$ \\
\hline$x \pm 14$ & $(8,41)$ \\
\hline$x \pm 20$ & $(2,23)$ \\
\hline$x \pm 30$ & $(6,44),(16,29),(30,24),(40,24),(120,24)$ \\
\hline$x \pm 56$ & $(2,47)$ \\
\hline$x \pm 70$ & $(112,48)$ \\
\hline$x \pm 150, x^{2} \pm 780$ & $(16,24)$ \\
\hline
\end{tabular}

Thus the exceptions in Theorem 1 are necessary.

Next we consider $L_{n}^{(\alpha)}(x)$ and more generally $\psi_{n}^{(\alpha)}(x)$ when $\alpha$ is a rational number with denominator 2. Thus $\alpha=u+\frac{1}{2}$ where $u$ is an integer. We recall that Hermite polynomials $H_{2 n}(x)$ and $H_{2 n+1}(x)$ are given by

$$
H_{2 n}(x)=(-1)^{n} 2^{2 n} n ! L_{n}^{\left(-\frac{1}{2}\right)}\left(x^{2}\right) \text { and } H_{2 n+1}(x)=(-1)^{n} 2^{2 n+1} n ! x L_{n}^{\left(\frac{1}{2}\right)}\left(x^{2}\right) .
$$

Schur [15], 14] proved that $L_{n}^{\left(-\frac{1}{2}\right)}\left(x^{2}\right)$ and $L_{n}^{\left(\frac{1}{2}\right)}\left(x^{2}\right)$ are irreducible and this implies the irreducibility of $H_{2 n}(x)$ and $H_{2 n+1}(x) / x$. We observe that $u \in\{-1,0\}$ in these results. Further, Laishram, Nair and Shorey [9] showed that $L_{n}^{(\alpha)}\left(x^{2}\right)$ with $\alpha=u+\frac{1}{2}$ and $1 \leq u \leq 45$ are irreducible except when $(u, n)=(10,3)$. In such a case $L_{3}^{\left(\frac{21}{2}\right)}\left(x^{2}\right)=$ $\frac{-1}{48}\left(2 x^{2}-15\right)\left(4 x^{4}-132 x^{2}+1035\right)$. It follows immediately from the above results that $L_{n}^{(\alpha)}(x)$ with $\alpha=u+\frac{1}{2}$ and $-1 \leq u \leq 45$ are irreducible except when $(u, n)=(10,3)$ where it has a linear factor. Further in the next result, we compute the Galois group of $L_{n}^{(\alpha)}(x)$ when $\alpha=u+\frac{1}{2}$. We prove

Theorem 2. Let $\alpha=u+\frac{1}{2}$ and $-1 \leq u \leq 45$. Then the Galois group of $L_{n}^{(\alpha)}(x)$ is $S_{n}$ except when $(u, n)=(10,3)$ in which case the Galois group is $\mathbb{Z}_{2}$.

Laishram [10] proved that the Galois group is $S_{n}$ when $u \in\{-1,0\}$. Therefore we assume that $u \geq 1$ in the proof of Theorem 2, By putting $a=1, d=2$ in (2), we have

$$
\psi_{n}^{(\alpha)}(x)=\sum_{j=0}^{n} a_{j}\left(\begin{array}{l}
n \\
j
\end{array}\right)(1+2(u+n)) \cdots(1+2(u+j+1)) x^{j} .
$$

It follows from the results of Schur on $G_{n}^{(\alpha)}(x)$ stated in the next section before Lemma 2.4 that $\psi_{n}^{(\alpha)}\left(x^{2}\right)$ with $\left|a_{0}\right|=\left|a_{n}\right|=1$ is irreducible when $u=-1$ and also $u=0$ unless $2 n+1$ is a power of 3 where it may have a linear factor or quadratic factor. Let

$\Omega_{1}=\left\{(2,2),(2,8),\left(2,2^{9}\right),\left(6,2^{4}\right),(9,4),\left(9,2^{6}\right),(10,3),(10,12),(10,24),(10,192),(16,8)\right.$ $\left.\left(21,2^{4}\right),\left(24,2^{4}\right),\left(30,2^{6}\right),\left(35,2^{5}\right),\left(35,2^{9}\right),(37,12),(37,36),(37,144),\left(44,2^{12}\right)\right\}$. 
Theorem 3. Let $\alpha=u+\frac{1}{2}$ where $1 \leq u \leq 45$ is an integer. Then $\psi_{n}^{(\alpha)}\left(x^{2}\right)$ with $\left|a_{0} a_{n}\right|=1$ is irreducible except when $(u, n) \in \Omega_{1}$ where it may have a quadratic factor or $(u, n)=(9,4)$ where it may have a factor of degree 4 . Further for every $(u, n) \in \Omega_{1}$ except for $(u, n)=\left(44,2^{12}\right)$, there exist integers $a_{0}, a_{1}, \ldots, a_{n}$ with $\left|a_{0}\right|=\left|a_{n}\right|=1$ such that $\psi_{n}^{(\alpha)}\left(x^{2}\right)$ has a quadratic factor. The quadratic factors are given in the following table.

TABLE 2

\begin{tabular}{|c|c|}
\hline$x^{2} \pm b$ & $(u, n)$ \\
\hline$x^{2} \pm 3$ & $(9,4),(10,3),\left(24,2^{4}\right)$ \\
\hline$x^{2} \pm 15$ & $\left(6,2^{4}\right),(10,12),(10,192),\left(21,2^{4}\right),\left(35,2^{5}\right)$ \\
\hline$x^{2} \pm 21$ & $(2,2),(2,8),\left(2,2^{9}\right),\left(9,2^{6}\right),\left(30,2^{6}\right),(37,36)$ \\
\hline$x^{2} \pm 33$ & $(37,12),(37,144)$ \\
\hline$x^{2} \pm 69$ & $(10,24)$ \\
\hline$x^{2} \pm 1095$ & $\left(35,2^{9}\right)$ \\
\hline$x^{2} \pm 7$ & $(16,8)$ \\
\hline
\end{tabular}

We have not been able to find a factorization for $(u, n)=\left(44,2^{12}\right)$ since $n$ is very large. We observe that the irreducibility of $\psi_{n}^{(\alpha)}\left(x^{2}\right)$ implies the irreducibility of $\psi_{n}^{(\alpha)}(x)$.

Corollary 1.1. Let $\alpha=u+\frac{1}{2}$ where $1 \leq u \leq 45$ is an integer. Then $\psi_{n}^{(\alpha)}(x)$ with $\left|a_{0} a_{n}\right|=1$ is irreducible except when $(u, n) \in \Omega_{1}$ where it may have a linear factor or $(u, n)=(9,4)$ where it may have a quadratic factor. Further for every $(u, n) \in \Omega_{1}$ except for $(u, n)=\left(44,2^{12}\right)$, there exist integers $a_{0}, a_{1}, \ldots, a_{n}$ with $\left|a_{0}\right|=\left|a_{n}\right|=1$ such that $\psi_{n}^{(\alpha)}(x)$ has a linear factor. The linear factors are obtained from the above table with $x^{2}$ replaced by $x$.

\section{Preliminaries}

The proofs of Theorems 1 and 3 depend on Newton polygons which we introduce now. Let $f(x)=\sum_{j=0}^{n} a_{j} x^{j} \in \mathbb{Z}[x]$ with $a_{0} a_{m} \neq 0$ and $p$ be a prime number. Let $S$ be the set of points in the extended plane

$$
S=\left\{\left(0, \nu\left(a_{m}\right)\right),\left(1, \nu\left(a_{m-1}\right)\right),\left(2, \nu\left(a_{m-2}\right)\right), \ldots,\left(m, \nu\left(a_{0}\right)\right)\right\}
$$

where for an integer $r$, we write $\nu(r)=\nu_{p}(r)$ for the highest power of $p$ dividing $r$ and we put $\nu(0)=\infty$. Consider the lower edges along the convex hull of these points. The left most endpoint is $\left(0, \nu\left(a_{m}\right)\right)$ and the right most endpoint is $\left(m, \nu\left(a_{0}\right)\right)$. The endpoints of each edge belong to $\mathrm{S}$ and the slopes of the edges increase from left to right. When referring to the edges of a Newton polygon, we shall not allow two different edges to have the same slope. The polygonal path formed by these edges is called the Newton polygon of $f(x)$ with respect to the prime $p$ and we denote it by $N P_{p}(f)$. The endpoints of the edges on $N P_{p}(f)$ are called the vertices of $N P_{p}(f)$. 
By a lattice point on an edge, we mean a lattice point on the edge other than the vertices of the edge. We denote the Newton function of $f$ with respect to the prime $p$ as the real function $f_{p}(x)$ on the interval $[0, m]$ which has the polygonal path formed by these edges as its graph. Hence $f_{p}(i)=\nu\left(a_{m-i}\right)$ for $i=0, m$ and at all points $i$ such that $\left(i, \nu\left(a_{m-i}\right)\right)$ is a vertex of $N P_{p}(f)$. We need the following result of Dumas [2].

Lemma 2.1. Let $g(x)$ and $h(x)$ be in $\mathbb{Z}[x]$ with $g(0) h(0) \neq 0$ and let $p$ be a prime. Let $k$ be a non-negative integer such that $p^{k}$ divides the leading coefficient of $g(x) h(x)$ but $p^{k+1}$ does not. Then the edges of the Newton polygon for $g(x) h(x)$ with respect to $p$ can be formed by constructing a polygonal path beginning at $(0, k)$ and using translates of the edges in the Newton polygons for $g(x)$ and $h(x)$ with respect to the prime $p$, using exactly one translate for each edge of the Newton polygons for $g(x)$ and $h(x)$. Necessarily, the translated edges are translated in such a way as to form a polygonal path with the slopes of the edges increasing.

Now we state a lemma of Filaseta [3] which is derived from Lemma 2.1.

Lemma 2.2. Let $l, k, m$ be integers with $m \geq 2 k>2 l \geq 0$. Suppose $g(x)=\sum_{j=0}^{m} b_{j} x^{j} \in$ $\mathbb{Z}[x]$ and $p$ be a prime such that $p \nmid b_{m}$ and $p \mid b_{j}$ for $0 \leq j \leq m-l-1$ and the right most edge of the $N P_{p}(g)$ has slope $<\frac{1}{k}$. Then for any integers $a_{0}, a_{1}, \ldots, a_{m}$ with $p \nmid a_{0} a_{m}$, the polynomial $f(x)=\sum_{j=0}^{m} a_{j} b_{j} x^{j}$ cannot have a factor with degree in $[l+1, k]$

Next we state some earlier results on polynomials which are more general than $\psi_{n}^{(\alpha)}(x)$. When $\alpha$ is an integer, the polynomials $\psi_{n}^{(\alpha)}(x)$ are a special case of the following class of polynomials first considered by Schur. Let $n \geq 1, a \geq 0$ and $a_{0}, a_{1}, \ldots, a_{n}$ be integers. The generalized Schur polynomials are defined as

$$
f_{n}^{(\alpha)}(x):=f_{n}^{(\alpha)}\left(x ; a_{0}, a_{1}, \cdots, a_{n}\right)=\sum_{j=0}^{n} a_{j} \frac{x^{j}}{(j+\alpha) !} .
$$

We observe that $(n+\alpha) ! f_{n}^{(\alpha)}(x)=\psi_{n}^{(\alpha)}(x)$ if $a_{j}$ are replaced by $a_{j}\left(\begin{array}{c}n \\ j\end{array}\right)$ in the definition of $f_{n}^{(\alpha)}(x)$.

Schur [14], [15] proved that $f_{n}^{(\alpha)}(x)$ with $\alpha \in\{0,1\}$ and $\left|a_{0} a_{n}\right|=1$ is irreducible unless $\alpha=1$ and $n+1=2^{r}$ for some $r$ where it may have a linear factor or $n=8$ where it may have a quadratic factor. Also for $\alpha=2$ and many other values of $\alpha$ the polynomial $f_{n}^{(\alpha)}(x)$ may have a linear factor. Laishram and Shorey [8] proved that

Lemma 2.3. Let $2 \leq k \leq \frac{n}{2}$ and $a_{0}, a_{1}, \ldots a_{n} \in \mathbb{Z}$ with $\left|a_{0} a_{n}\right|=1$. Asssume that $0 \leq \alpha \leq 40$ if $k=2$ and $0 \leq \alpha \leq 50$ if $k>2$. Then $f_{n}^{(\alpha)}(x)$ has no factor of degree $k$ 
except possibly when $(n, k, \alpha)$ is given by

$$
\begin{aligned}
& k=3,(n, \alpha) \in\{(7,3),(8,2),(12,4),(46,4),(14,12),(17,11),(53,12)\} \\
& k=4,(n, \alpha) \in\{(18,9),(18,10),(56,10),(16,12),(17,11),(38,13),(39,18)\} \\
& k=5,(n, \alpha) \in\{(17,11),(19,9),(40,12)\}
\end{aligned}
$$

and $k=2$ with $(n, \alpha)$ satisfying

(i) $n+\alpha \leq 100$

(ii) $\alpha \in\{13,14,19,33\}, n+\alpha \in\{126,225,2401,4375\}$

(iii) $(n, \alpha) \in\{(112,9),(233,10),(234,9)\}$

together with the following set of pairs $(n, \alpha)$ given by the table:

TABLE 3

\begin{tabular}{|c|c||c|c||c|c|}
\hline$\alpha$ & $n+\alpha$ & $\alpha$ & $n+\alpha$ & $\alpha$ & $n+\alpha$ \\
\hline 12 & 169,729 & 15,16 & 289 & 17 & 513 \\
\hline 18 & $361,513,1216$ & 19,20 & 243 & 21 & 529 \\
\hline 21,22 & 121,576 & 24 & $325,625,676$ & 27 & 784 \\
\hline 28 & 145 & 29 & 961 & 31 & 243 \\
\hline 32 & $243,289,1089$ & 33 & $136,256,289,5832$ & 36 & 1369 \\
\hline 38 & $325,625,676$ & 39 & 1025,6561 & 40 & 288 \\
\hline
\end{tabular}

The above result on $f_{n}^{(\alpha)}(x)$ has a large number of exceptions especially when $k=2$. Moreover it gives no information on linear factors. Hence in this paper, we consider $\psi_{n}^{(\alpha)}(x)$ which is a special case of $f_{n}^{(\alpha)}(x)$, but more general than $L_{n}^{(\alpha)}(x)$ and we get complete irreducibility results for $\psi_{n}^{(\alpha)}(x)$. Analogously we consider the polynomial $G_{n}^{(\alpha)}(x)$ which is more general than $\psi_{n}^{(\alpha)}(x)$. For integers $a_{0}, a_{1}, \ldots, a_{n}$ and $\alpha$ given by (1), let

$$
G_{n}^{(\alpha)}(x)=G_{n}^{(\alpha)}\left(x ; a_{0}, a_{1}, \ldots, a_{n}\right)=\sum_{j=0}^{n} a_{j}(n+\alpha)(n-1+\alpha) \cdots(j+1+\alpha) d^{n-j} x^{j}
$$

We observe that

$$
(n+\alpha) ! f_{n}^{(\alpha)}(x)=G_{n}^{(\alpha)}(x) \text { when } \alpha \text { is an integer. }
$$

Let $\alpha$ be a rational with denominator 2 . Then by (11), $\alpha=u+\frac{1}{2}$ and

$$
G_{n}^{(\alpha)}(x)=\sum_{j=0}^{n} a_{j} x^{j}\left(\prod_{i=j+1}^{n}(1+2(u+i))\right)
$$

Schur [14], [15] proved that $G_{n}^{(\alpha)}\left(x^{2}\right)$ with $\left|a_{0}\right|=\left|a_{n}\right|=1$ is irreducible when $u \in$ $\{-1,0\}$ unless $u=0$ and $2 n+1$ is a power of 3 where it may have a linear or quadratic factor. Let $A=\left\{ \pm 2^{t}: t \geq 0, t \in \mathbb{Z}\right\}$ and $S=\{(1,121),(8,59),(8,114),(9,4),(9,113)$, $(9,163),(9,554),(15,23),(15,107),(16,106),(20,102),(21,101),(26,155),(26,287),(30,92)$, 
$(36,86),(43,1158),(44,716)\}$. Laishram, Nair and Shorey [9] proved the following irreducibility results on $G_{n}^{(\alpha)}\left(x^{2}\right)$. We observe that in [9] the polynomials $G_{n}^{(\alpha)}(x)$ are denoted by $G_{\alpha}(x)$.

Lemma 2.4. Let $1 \leq u \leq 45$ and $\alpha=u+\frac{1}{2}$. Let $a_{0}, a_{n} \in A$. Then $G_{n}^{(\alpha)}\left(x^{2}\right)$ has no factor of degree $\geq 3$ except where $(u, n) \in\{(1,12),(6,7),(9,113),(10,3),(21,101)\}$ or $(u, n) \in S$ or $(u, n)=(44,79)$ where it may have a factor of degree 3 or 4 or 6 , respectively.

The proof for the irreducibility of $L_{n}^{(\alpha)}\left(x^{2}\right)$ given in Section 7 of [9] based on Newton polygons is also valid for $\psi_{n}^{(\alpha)}\left(x^{2}\right)$ when $\alpha=u+\frac{1}{2}$ except for the pairs $(u, n) \in T_{0}$ where it may have a linear or quadratic factor where

$$
\begin{aligned}
T_{0}= & (2,2),(2,8),\left(2,2^{9}\right),\left(6,2^{4}\right),(9,4),\left(9,2^{6}\right),(10,3),(10,12),(10,24),(10,192),(11,2), \\
& \left(16,2^{3}\right),\left(21,2^{4}\right),\left(24,2^{4}\right),\left(30,2^{6}\right),(35,2),\left(35,2^{5}\right),\left(35,2^{9}\right),\left(36,2^{6}\right),(37,12),(37,36), \\
& \left.(37,144),(38,2),\left(44,2^{12}\right)\right\} .
\end{aligned}
$$

For $(u, n) \in T_{0}$, we have computed $L_{n}^{(\alpha)}\left(x^{2}\right)$ in [9] to find that it is irreducible except at $(u, n)=(10,3)$. But in this case of $\psi_{n}^{(\alpha)}\left(x^{2}\right)$, since $a_{j}$ 's are arbitrary, we cannot exclude these pairs as we did it for $L_{n}^{(\alpha)}\left(x^{2}\right)$. Thus we have

Lemma 2.5. Let $1 \leq u \leq 45$ and $\alpha=u+\frac{1}{2}$. Then $\psi_{n}^{(\alpha)}\left(x^{2}\right)$ with $\left|a_{0} a_{n}\right|=1$ has no factor of degree in $\{1,2\}$ except when $(u, n) \in T_{0}$.

Lemma 2.6. The diophantine equation

$$
x+y=z
$$

in $x, y, z \in S=\left\{2^{x_{1}} \cdots 13^{x_{6}} ; x_{i} \in \mathbb{Z}, x_{i} \geq 0\right\}$ with $\operatorname{gcd}(x, y)=1$ and $x \leq y$ has exactly 545 solutions. Out of them 514 satisfy $\operatorname{ord}_{2}(x y z) \leq 12, \operatorname{ord}_{3}(x y z) \leq 7, \operatorname{ord}_{5}(x y z) \leq 5$, $\operatorname{ord}_{7}(x y z) \leq 4, \operatorname{ord}_{11}(x y z) \leq 3, \operatorname{ord}_{13}(x y z) \leq 3$. The remaining 31 solutions are given in [17, Table IX].

This is due to de Weger [17]. Further we need the following result from [4, Lemma 4.1] which is a direct application of Lemma 2.1 for determining $a_{0}, a_{1}, \ldots, a_{n}$ such that $\psi_{n}^{(\alpha)}(x)$ has a linear factor when $(n, \alpha) \in \Omega$.

Lemma 2.7. Let $w(x)$ be a monic polynomial in $\mathbb{Z}[x]$ divisible by $x-b$ with $b \in \mathbb{Z}$. Let $p$ be a prime and e be a non-negative integer for which $p^{e} \| b$. Then $N P_{p}(w(x))$ with respect to $p$ has an edge that includes a translate of the line segment joining $(0,0)$ to $(1, e)$. Also, if the right most edge has slope $<1$, then necessarily $e=0$.

Lemma 2.8. Let $p$ be a prime. For any integer $l \geq 1$, write $l$ in base $p$ as $l=$ $l_{t} p^{t}+l_{t-1} p^{t-1}+\cdots+l_{1} p+l_{0}$ where $0 \leq l_{i} \leq p-1$ for $0 \leq i \leq t$ and $l_{t}>0$. Then

$$
\nu_{p}(l !)=\frac{l-\sigma_{p}(l)}{p-1}
$$

where $\sigma_{p}(l)=l_{t}+l_{t-1}+\cdots+l_{1}+l_{0}$. 
This is due to Legendre. For a proof, see [7, Ch.17, p 263].

Lemma 2.9. Let $r \in\{1,3\}$. The interval $(x, 1.048 x]$ contain primes congruent to $r$ modulo 4 when $x \geq 887$.

This follows from [1, Theorem 1] with $k=4$.

\section{Lemmas For the PROOF of TheOrem 1}

Let $\alpha$ be an integer throughout this section. We write

$$
\Delta_{j}=\Delta(\alpha+1, j)=(\alpha+1)(\alpha+2) \cdots(\alpha+j) .
$$

For the proof of Theorem 1, we need the following result which is an analogous for $f_{n}^{(\alpha)}(x)$ as proved in [8, Lemma 1.1].

Lemma 3.1. Let $\alpha>0,1 \leq k \leq \frac{n}{2}$ and $u_{0}=\frac{\alpha}{k}$. Assume that there is a prime $p \geq k+2$ with

$$
p \mid \prod_{i=1}^{k}(n-k+i)(\alpha+n-k+i), \quad p \nmid a_{0} a_{n} \prod_{i=1}^{k}(\alpha+i) .
$$

Suppose

$$
p \geq \min \left(2 u_{0}, k+u_{0}\right)
$$

or

$$
p>2 k \text { and } p^{2}-p \geq \alpha .
$$

Then $\psi_{n}^{(\alpha)}(x)$ has no factor of degree $k$.

The proof of Lemma 3.1 is exactly same as the proof of [8, Lemma 1.1] for $f_{n}^{(\alpha)}(x)$. Further we prove the following result analogus to Lemma 3.1 with $k \in\{1,2\}$.

Lemma 3.2. Let $k \in\{1,2\}$ and $p \geq 2 k+1$ be such that $p \mid \prod_{i=1}^{k}(n-k+i), 0<\alpha \leq 50$ and

$$
\nu_{p}((\alpha+1) \cdots(\alpha+j)) \leq \nu_{p}(n(n-1) \cdots(n-j+1)) \text { for } 1 \leq j \leq k .
$$

Then $\psi_{n}^{(\alpha)}(x)$ with $\left|a_{0} a_{n}\right|=1$ has no factor of degree $k$ except when $k=1, p=3, \alpha \in$ $\{24,25\}$ and $\nu_{3}(n)=1$.

Proof. We use Lemma 2.2 with $g(x)=g_{n}^{(\alpha)}(x), m=n$, and $l=k-1$ where

$$
g_{n}^{(\alpha)}(x)=\sum_{j=0}^{n}\left(\begin{array}{l}
n \\
j
\end{array}\right)(n+\alpha) \cdots(j+1+\alpha) x^{j} .
$$

We observe that $b_{j}=\left(\begin{array}{c}n \\ j\end{array}\right) \frac{(\alpha+n) !}{(\alpha+j) !}$. For $0 \leq j \leq n-p$, we see that $p \mid \frac{(\alpha+n) !}{(\alpha+j) !}$ since a product of $p$ consecutive positive integers is divisible by $p$. Let $n-p<j \leq n-k$. Then 
$k \leq n-j<p$ and $\left(\begin{array}{c}n \\ j\end{array}\right)=\frac{n(n-1) \cdots(j+1)}{(n-j) !}$. Therefore $p \mid\left(\begin{array}{c}n \\ j\end{array}\right)$ since $p \mid n(n-1) \cdots(n-k+1)$. Hence $p \mid b_{j}$ for $0 \leq j \leq n-k$. Therefore it suffices to show that

$$
\frac{\nu_{p}\left(\Delta_{j}\right)-\nu_{p}\left(\left(\begin{array}{c}
n \\
j
\end{array}\right)\right)}{j}<\frac{1}{k} \text { for } 1 \leq j \leq n .
$$

Clearly this is true for $j=1,2, \cdots, k$ by our assumption. Hence we take $j>k$. Since $\Delta_{j}=\frac{(\alpha+j) !}{\alpha !}$, we have by Lemma 2.8

$$
\frac{\nu_{p}\left(\Delta_{j}\right)}{j}=\frac{j-\sigma(\alpha+j)+\sigma(\alpha)}{(p-1) j}=\frac{1}{p-1}+\frac{\sigma(\alpha)-\sigma(\alpha+j)}{(p-1) j} \text { for } 1 \leq j \leq n .
$$

Let $j \geq \alpha$. Since $p \geq 2 k+1, \sigma(\alpha) \leq \alpha$ and $\sigma(\alpha+j) \geq 1$, we have

$$
\frac{\nu_{p}\left(\Delta_{j}\right)}{j} \leq \frac{1}{2 k}+\frac{\alpha-1}{2 k j} \leq \frac{1}{2 k}+\frac{\alpha-1}{2 k \alpha}<\frac{1}{k} .
$$

Hence we may suppose that $j<\alpha \leq 50$. Then $\alpha+j \leq 2 \alpha-1 \leq 99$. Let $1 \leq j_{0} \leq j$ be such that $\max _{1 \leq i \leq j} \nu_{p}(\alpha+i)=\nu_{p}\left(\alpha+j_{0}\right):=\nu_{0}$. Then

$$
\begin{aligned}
\frac{\nu_{p}\left(\Delta_{j}\right)-\nu_{p}\left(\left(\begin{array}{l}
n \\
j
\end{array}\right)\right)}{j} & \leq \frac{\nu_{p}\left(\alpha+j_{0}\right)+\nu((j-1) !)-\nu_{p}\left(\left(\begin{array}{c}
n \\
j
\end{array}\right)\right)}{j} \\
& \leq \frac{\nu_{0}+\frac{j-2}{p-1}-\nu_{p}\left(\left(\begin{array}{c}
n \\
j
\end{array}\right)\right)}{j}
\end{aligned}
$$

using Lemma 2.8. Since $p \geq 2 k+1, \frac{j-2}{(p-1) j} \leq \frac{j-2}{2 k j}=\frac{1}{2 k}-\frac{1}{k j}$ and therefore

$$
\frac{\nu_{p}\left(\Delta_{j}\right)-\nu_{p}\left(\left(\begin{array}{c}
n \\
j
\end{array}\right)\right)}{j} \leq \frac{\nu_{0}-\frac{1}{k}-\nu_{p}\left(\left(\begin{array}{c}
n \\
j
\end{array}\right)\right)}{j}+\frac{1}{2 k} \text {. }
$$

Then $\frac{\nu_{p}\left(\Delta_{j}\right)-\nu_{p}\left(\left(\begin{array}{c}n \\ j\end{array}\right)\right)}{j}<\frac{1}{k}$ if $j>2 \nu_{0} k-2-2 k \nu_{p}\left(\left(\begin{array}{c}n \\ j\end{array}\right)\right)$. Hence we now suppose $j \leq$ $2 \nu_{0} k-2-2 k \nu_{p}\left(\left(\begin{array}{l}n \\ j\end{array}\right)\right)$.

Let $k=2$. Then $p \geq 5$. Since $\alpha+j \leq 99$, we have $\nu_{0} \leq 2$. Hence $j \leq 6-4 \nu_{p}\left(\left(\begin{array}{c}n \\ j\end{array}\right)\right)$. Further $j \geq 3$ since $j>k$. Hence $3 \leq j \leq 6-4 \nu_{p}\left(\left(\begin{array}{c}n \\ j\end{array}\right)\right)$ implying $j \leq 6$ and $\nu_{p}\left(\left(\begin{array}{c}n \\ j\end{array}\right)\right)=0$. Since $p \mid\left(\begin{array}{c}n \\ j\end{array}\right)$ for $2 \leq j<p$, we have $p=5$ and $j \in\{5,6\}$. Further we have from $\alpha \leq 50$ that $\frac{\nu_{5}\left(\Delta_{5}\right)}{5} \leq \frac{2}{5}<\frac{1}{2}$ giving (8). Hence we need to consider only $j=6$ and it suffices to show that

$$
\frac{\nu_{5}\left(\Delta_{6}\right)-\nu_{5}\left(\left(\begin{array}{l}
n \\
6
\end{array}\right)\right)}{6}<\frac{1}{2} .
$$

If $5 \nmid(\alpha+1)$, then $\nu_{5}\left(\Delta_{j}\right) \leq 2$ and we are done. Hence $5 \mid(\alpha+1)$. Since $\nu_{p}(\alpha+1) \leq \nu_{p}(n)$ by our assumption, we have $5 \mid n$ and further

$$
\begin{aligned}
\frac{\nu_{5}\left(\Delta_{6}\right)-\nu_{5}\left(\left(\begin{array}{l}
n \\
6
\end{array}\right)\right)}{6} & =\frac{\nu_{5}(\alpha+1)+\nu_{5}(\alpha+6)-\nu_{5}(n)-\nu_{5}(n-5)+1}{6} \\
& \leq \frac{\left.\nu_{5}(\alpha+6)\right)-\nu_{5}(n-5)+1}{6} \leq \frac{\left.\nu_{5}(\alpha+6)\right)}{6} \leq \frac{2}{6}<\frac{1}{2}
\end{aligned}
$$

since $5 \mid(n-5)$ and $\alpha+6 \leq 56$. 
Let $k=1$. Then $j \leq 2 \nu_{0}-2-2 \nu_{p}\left(\left(\begin{array}{l}n \\ j\end{array}\right)\right)$. Let $\nu_{0} \leq 2$. Then $j \leq 2-2 \nu_{p}\left(\left(\begin{array}{l}n \\ j\end{array}\right)\right)$. Recall that $j \geq 2$ and hence $j=2$ and $\nu_{p}\left(\left(\begin{array}{c}n \\ 2\end{array}\right)\right)=0$ which is not possible since $p \geq 3, p \mid n$. Thus $\nu_{0} \geq 3$. Then $p=3$. Further from $\alpha+j \leq 99$ we get $\nu_{0} \leq 4$ implying $j \leq 6$ which together with $\alpha \leq 50$ gives $\nu_{0}=3$ and hence $j \leq 4-2 \nu_{p}\left(\left(\begin{array}{l}n \\ j\end{array}\right)\right)$. In particular, $j \leq 4$. When $j=4$, we have $3 \mid\left(\begin{array}{l}n \\ 4\end{array}\right)$ and $\nu_{3}\left(\Delta_{4}\right) \leq 4$ and hence the assertion (8) is valid. Thus $j \in\{2,3\}$. This, together with $\nu_{0}=3$ and $j_{0} \leq j$, implies $\alpha+j_{0}=27$ and $\nu_{3}\left(\alpha+j_{0}\right)=3$. Further $\nu_{3}\left(\Delta_{j}\right)=\nu_{3}\left(\alpha+j_{0}\right)$ since $j \in\{2,3\}$. Therefore we may assume $3-\nu_{3}\left(\left(\begin{array}{l}n \\ j\end{array}\right)\right) \geq j$ if $j=2,3$ else (8) is valid. Let $j=2$. Then $\nu_{3}\left(\left(\begin{array}{l}n \\ 2\end{array}\right)\right)=\nu_{3}(n) \geq 1$ implying $\nu_{3}(n)=1$. We see that $j_{0} \neq 1$, otherwise $3=\nu_{3}(\alpha+1) \leq \nu_{3}(n)$ by our assumption and this is not possible. Thus $j_{0}=2$ implying $\alpha=25$. Let $j=3$. Then $\nu_{3}\left(\left(\begin{array}{l}n \\ 3\end{array}\right)\right)=\nu_{3}(n)-1$. Hence we can assume that $\nu_{3}(n)=1$ and $j_{0} \neq 1$ as in the above case. This give $\alpha \in\{24,25\}$ and $\nu_{3}(n)=1$.

Let

$$
S_{M}=\{n \geq 1: n, P(n(n+1)) \leq M\} .
$$

The sets $S_{M}$ for $M \leq 41$ are given by tables in Lehmer [11, Table IIA] and for $M=100$ by table in Luca and Najman [12] and [13].

Lemma 3.3. Let $k=2$ and $40<\alpha \leq 50$. Then $\psi_{n}^{(\alpha)}(x)$ has no factor of degree 2 .

Proof. Assume that $\psi_{n}^{(\alpha)}(x)$ has a factor of degree 2. If $P(n(n-1)(n+\alpha)(n+\alpha-1)) \geq$ 53 , then $\psi_{n}^{(\alpha)}(x)$ has no factor of degree 2 by Lemma 3.1. Hence we may assume that $P(n(n-1)(n+\alpha)(n+\alpha-1)) \leq 47$. We refer to the tables of [12] to find $n$ and $\alpha$ such that $P(n(n-1)(n+\alpha)(n+\alpha-1)) \leq 47$. For these pairs $(n, \alpha)$, we find a prime $p$ to apply Lemmas 3.1 and 3.2 to conclude that $\psi_{n}^{(\alpha)}(x)$ has no factor of degree 2 except for pairs $(n, \alpha) \in\{(4,45),(4,46),(6,44),(8,41),(9,41),(12,43),(16,48)\}$. Let $(n, \alpha)=(12,43)$. Then $N P_{3}\left(g_{n}^{(\alpha)}(x)\right)$ where $g_{n}^{(\alpha)}(x)$ is given by (7) has vertices

$$
\{(0,0),(9,5),(12,7)\} \text {. }
$$

We derive the different possibilities for $N P_{3}\left(\psi_{n}^{(\alpha)}(x)\right)$ using $N P_{3}\left(g_{n}^{(\alpha)}(x)\right)$. If $3 \mid a_{3}$, then the vertices of $N P_{3}\left(\psi_{n}^{(\alpha)}(x)\right)$ are given by $\{(0,0),(12,7)\}$. Hence by Lemma 2.1, $\psi_{n}^{(\alpha)}(x)$ has no factor of degree 2. If $3 \nmid a_{3}$, then the vertices of $N P_{3}\left(\psi_{n}^{(\alpha)}(x)\right)$ is same as $N P_{3}\left(g_{n}^{(\alpha)}(x)\right)$. Again by Lemma 2.1, $\psi_{n}^{(\alpha)}(x)$ has no factor of degree 2. Now we apply Lemma 2.2 with the following choice of primes for each of the other values of $n$ and $\alpha$ to conclude that $\psi_{n}^{(\alpha)}(x)$ has no factor of degree 2 .

\begin{tabular}{|c|c|}
\hline$p$ & $(n, \alpha)$ \\
\hline 2 & $(4,45),(16,48)$ \\
\hline 3 & $(4,46),(9,41)$ \\
\hline 7 & $(6,44),(8,41)$ \\
\hline
\end{tabular}


Denote by $T$ the set of all triplets $(n, \alpha, k)$ listed in Lemma 2.3. Further we put $T_{1}:=\{(8,13,2),(6,19,2),(9,19,2),(8,20,2),(4,21,2),(12,21,2),(24,22,2),(16,24,2),(9,27,2)$, $(18,33,2),(16,34,2),(9,40,2),(27,38,2),(14,12,3),(16,12,4)\}$. We observe that $T_{1}$ is a subset of $T$.

Lemma 3.4. Let $2 \leq k \leq \frac{n}{2}$ and $11 \leq \alpha \leq 50$. Then $\psi_{n}^{(\alpha)}(x)$ has no factor of degree $k$ except for $(n, \alpha, k)=(16,24,2)$.

Proof. Assume that $\psi_{n}^{(\alpha)}(x)$ has a factor of degree $k$. By Lemma 3.3, we may assume that $\alpha \leq 40$ when $k=2$. Since the irreducibility of $f_{n}^{(\alpha)}(x)$ implies the irreducibilty of $\psi_{n}^{(\alpha)}(x)$, we may assume, by Lemma 2.3, that $\psi_{n}^{(\alpha)}(x)$ has no factor of degree $k \geq 2$ except for the triplets $(n, \alpha, k) \in T$. We consider $(n, \alpha, k)=(7,2,2) \in T$. Here $p=7$ divides $n$ but does not divide $(\alpha+1)(\alpha+2)=12$ and $p>2 k$ and $p^{2}-p \geq \alpha$. Now we derive from Lemma 3.1 that $\psi_{n}^{(\alpha)}(x)$ has no factor of degree 2. For $(n, \alpha, k)=$ $(6,3,2) \in T$, we are not able to find a prime $p$ satisfying Lemma 3.1, but we apply Lemma 3.2 with $p=5$ to conclude that $\psi_{n}^{(\alpha)}(x)$ has no factor of degree 2 . We apply Lemmas 3.1 and 3.2 similarly to conclude that we are left with $(n, \alpha, k) \in T_{1}$ among triplets in $T$. Let $(n, \alpha, k) \in\{(14,12,3),(16,12,4)\}$. When $(n, \alpha, k)=(14,12,3)$, we have $N P_{7}\left(g_{n}^{(\alpha)}(x)\right)=\{(0,0),(14,2)\}$ and when $(n, \alpha, k)=(16,12,4)$, we have $N P_{2}\left(g_{n}^{(\alpha)}(x)\right)=\{(0,0),(16,15)\}$. Therefore these cases are excluded by Lemmas 2.2 and 2.1, respectively. We now take $(n, \alpha, k) \in T_{1}-\{(16,24,2)\}$ and may suppose that $k=2$. We calculate the Newton polygons for $g_{n}^{(\alpha)}(x)$ given by (7) in each of these cases with a suitable prime so that the conditions of Lemma 2.2 are satisfied. Then we calculate the slope of the right most edge in each case. If the slope of the right most edge is $<\frac{1}{2}$, we exclude it by Lemma 2.2 and the cases where the slope of the right most edge is $\geq \frac{1}{2}$ are excluded by applying Lemma 2.1. We illustrate this by some examples. Let $(n, \alpha)=(6,19)$. Then the vertices for $N P_{3}\left(g_{n}^{(\alpha)}(x)\right)$ are given by $\{(0,0),(6,2)\}$ and the slope of the right most edge is $\frac{1}{3}<\frac{1}{2}$. Hence $\psi_{n}^{(\alpha)}(x)$ does not have a factor of degree 2 by Lemma 2.2. Let $(n, \alpha)=(9,19)$. The vertices for $N P_{3}\left(g_{n}^{(\alpha)}(x)\right)$ are given by $\{(0,0),(9,5)\}$. Here $N P_{3}\left(g_{n}^{(\alpha)}(x)\right)$ is same as $N P_{3}\left(\psi_{n}^{(\alpha)}(x)\right)$ and the maximum slope is $\frac{5}{9}>\frac{1}{2}$. However $N P_{3}\left(\psi_{n}^{(\alpha)}(x)\right)$ has only one edge with lattice points $(0,0)$ and $(9,5)$. Hence $\psi_{n}^{(\alpha)}(x)$ has no factor of degree 2 by Lemma 2.1.

For each of the following pairs of $(n, \alpha)$, we give a choice of a prime $p$ for considering its Newton polygon and then we conclude as above that $\psi_{n}^{(\alpha)}(x)$ has no factor of degree 2 .

\begin{tabular}{|c|c|}
\hline$p$ & $(n, \alpha)$ \\
\hline 2 & $(8,20),(12,21),(16,34)$ \\
\hline 3 & $(4,21),(9,40),(18,33),(27,38)$ \\
\hline 5 & $(9,27),(24,22)$ \\
\hline
\end{tabular}

Now it remains to consider the pair $(n, \alpha)=(8,13)$. We calculate $N P_{7}\left(g_{n}^{(\alpha)}(x)\right)=$ $\{(0,0),(7,1),(8,2)\}$. We consider the possibilities for $N P_{7}\left(\psi_{n}^{(\alpha)}(x)\right)$. If $7 \mid a_{1}$, then 
$N P_{7}\left(\psi_{n}^{(\alpha)}(x)\right)=\{(0,0),(8,2)\}$ and if $7 \nmid a_{1}$, then $N P_{7}\left(\psi_{n}^{(\alpha)}(x)\right)=\{(0,0),(7,1),(8,2)\}$. In both cases it is clear from Lemma 2.1 that $\psi_{n}^{(\alpha)}(x)$ has no factor of degree 2.

Next we formulate a computational lemma.

Lemma 3.5. Let $11 \leq \alpha \leq 50$ and $2 \leq n \leq 50$. Then $\psi_{n}^{(\alpha)}(x)$ has no linear factor except for $(n, \alpha) \in \Omega$ with $n \leq 50$.

Proof. Assume that $\psi_{n}^{(\alpha)}(x)$ has a linear factor and $(n, \alpha) \notin \Omega$. First we consider $\alpha \in\{24,25\}$ and $\nu_{3}(n)=1$. Then $n \in\{3,6,12,15,21,24,30,33,39,42,48\}$. We exclude the pairs given by $\alpha=24, n \in\{15,21,33,39,42\}$ and $\alpha=25, n \in$ $\{3,6,12,21,24,30,33,42,48\}$ by Lemma 3.1. Let $(n, \alpha)=(3,24)$. We may assume that $\psi_{n}^{(\alpha)}(x)=x^{3}+81 a_{1} x^{2}+2106 a_{2} x \pm 17550$. Let $x-b$ be a linear factor for $\psi_{n}^{(\alpha)}(x)$. Then $b \mid 17550$. Since $17550=2 \cdot 3^{3} \cdot 5^{2} \cdot 13, b$ is composed of primes $\{2,3,5,13\}$ and $5^{3} \nmid b$. We consider the polynomial $g_{n}^{(\alpha)}(x)=x^{3}+81 x^{2}+2106 x+17550$. Then $N P_{2}\left(g_{n}^{(\alpha)}(x)\right)=\{(0,0),(1,0),(3,1)\}, N P_{3}\left(g_{n}^{(\alpha)}(x)\right)=\{(0,0),(3,3)\}, N P_{5}\left(g_{n}^{(\alpha)}(x)\right)=$ $\{(0,0),(1,0),(2,0),(3,2)\}, N P_{13}\left(g_{n}^{(\alpha)}(x)\right)=\{(0,0),(1,0),(3,1)\}$. Since the slope of the right most edge of $N P_{p}\left(\psi_{n}^{(\alpha)}(x)\right)$ is at most equal to that of $N P_{p}\left(g_{n}^{(\alpha)}(x)\right)$, we see that the slope of the right most edge of $N P_{2}\left(\psi_{n}^{(\alpha)}(x)\right)$ and that of $N P_{13}\left(\psi_{n}^{(\alpha)}(x)\right)<1$. Thus $2 \nmid b$, and $13 \nmid b$ by Lemma 2.7. Further $N P_{3}\left(\psi_{n}^{(\alpha)}(x)\right)=N P_{3}\left(g_{n}^{(\alpha)}(x)\right)$ and hence by Lemmas 2.1 and 2.7, we have $3 \| b$. Write $b=3 b_{1}$ with $b_{1} \in\left\{ \pm 1, \pm 5, \pm 5^{2}\right\}$. Then $\psi_{n}^{(\alpha)}(b)=0$ implies $3^{3}\left\{b_{1}^{3}+3^{3} a_{1} \pm 3^{2} \cdot 26 a_{2} \pm 650\right\}=0$. This gives $3^{2} \mid\left(b_{1}^{3} \pm 650\right)$ which is not true for $b_{1} \in\left\{ \pm 1, \pm 5, \pm 5^{2}\right\}$. Thus $\psi_{n}^{(\alpha)}(x)$ has no linear factor when $(n, \alpha)=(3,24)$. Now consider the pairs given by $\alpha=24, n=6$ and $\alpha=25, n \in$ $\{15,39\}$ since the remaining pairs are in $\Omega$. These pairs are excluded by Lemma 2.2 with $p=2,(n, \alpha)=(6,24) ; p=5,(n, \alpha)=(15,25)$ and $p=13,(n, \alpha)=(39,25)$. We may now assume that either $\alpha \in\{24,25\}, \nu_{3}(n) \neq 1$ or $\alpha \notin\{24,25\}$. All these pairs other than 52 pairs are excluded by Lemmas 3.1 or 3.2 and the 52 pairs are excluded by Lemmas 2.1 and 2.2 as in the proof of Lemma 3.4 .

Lemma 3.6. For $(n, \alpha) \in \Omega$, there exists $a_{0}, a_{1}, \ldots, a_{n} \in \mathbb{Z}$ with $\left|a_{0}\right|=\left|a_{n}\right|=1$ such that $\psi_{n}^{(\alpha)}(x)=\psi_{\alpha}\left(x ; a_{0}, a_{1}, \ldots, a_{n}\right)$ has a linear factor.

Proof. Let $(n, \alpha)=(40,24) \in \Omega$. Assume that $x-b$ is a factor of $\psi_{n}^{(\alpha)}(x)$. Then $b$ divides the constant term of $\psi_{n}^{(\alpha)}(x)$ given by $25 \cdot 26 \cdot 27 \cdots 64$. Let $p \geq 7$ be a prime dividing $25 \cdot 26 \cdot 27 \cdots 64$. We find that the slope of right most edge of $N P_{p}\left(g_{n}^{(\alpha)}(x)\right)<1$. Since the slope of the right most edge of $N P_{p}\left(\psi_{n}^{(\alpha)}(x)\right)$ is at most equal to that of $N P_{p}\left(g_{n}^{(\alpha)}(x)\right)$, we see the the slope of the right most edge of $N P_{p}\left(\psi_{n}^{(\alpha)}(x)\right)<1$. Thus $p \nmid b$ by Lemma 2.7. For $p \leq 5$, the details of vertices of $N P_{p}\left(g_{n}^{(\alpha)}(x)\right)$ are given below.

$$
\begin{aligned}
& N P_{2}\left(g_{n}^{(\alpha)}(x)\right)=\{(0,0),(32,32),(40,41)\} \\
& N P_{3}\left(g_{n}^{(\alpha)}(x)\right)=\{(0,0),(1,0),(10,4),(37,17),(40,20)\} \\
& N P_{5}\left(g_{n}^{(\alpha)}(x)\right)=\{(0,0),(10,2),(35,8),(39,9),(40,10)\} .
\end{aligned}
$$


In each of the above cases, $N P_{p}\left(g_{n}^{(\alpha)}(x)\right)$ has lattice points which give edges of length 1 and slope 1 . Here we consider $x+30$ as a possible linear factor. Equating the remainder obtained by dividing $\psi_{n}^{(\alpha)}(x)$ with $x+30$ to be 0 and solving the equation in integers, we get the values for $a_{j}^{\prime}$ s. If $x+30$ is a factor of $\psi_{n}^{(\alpha)}(x)$ then we observe that $x-30$ is a factor of $-\psi_{n}^{(\alpha)}(-x)$. The details of the linear factors for other pairs $(n, \alpha) \in \Omega$ are given in Table 1 .

Lemma 3.7. Let $P(n) \geq 3, \nu_{3}(n)=1, n>50$ and $\alpha \in\{24,25\}$. Then $\psi_{n}^{(\alpha)}(x)$ has no linear factor except when $(n, \alpha)=(120,24)$.

Proof. Let $\alpha=24$ and $\nu_{3}(n)=1$. By Lemma 3.1, we may assume that

$$
\text { if } p \mid n(n+\alpha) \text {, then either } p \mid(\alpha+1) \text { or } p^{2}-p<\alpha \text {. }
$$

Thus $P(n(n+24)) \leq 5$. By Lemma 3.2, we may assume that $\nu_{5}(n) \leq 1$. Thus we have

$$
\begin{aligned}
n & =2^{\alpha_{1}} 3 \cdot 5^{\gamma_{1}}, & & \gamma_{1} \leq 1 \\
n+24 & =2^{\alpha_{2}} 3^{\beta_{2}} 5^{\gamma_{2}}, & & \beta_{2} \geq 1
\end{aligned}
$$

where $\alpha_{1}, \alpha_{2}, \beta_{2}, \gamma_{1}, \gamma_{2}$ are non-negative integers. Let $\gamma_{1}=0$. Then $n=2^{\alpha_{1}} \cdot 3>50$ implies $\alpha_{1} \geq 5$. Thus $\alpha_{2}=\operatorname{ord}_{2}(n+24)=3$. Thus the above equations give

$$
3^{\beta_{2}-1} 5^{\gamma_{2}}-2^{\alpha_{1}-3}=1 \text {. }
$$

We solve this equation using Lemma 2.6. From now onwards, we solve the diophantine equation $x+y=z$ with $x \leq y, P(x y z) \leq 13$ and $\operatorname{gcd}(x, y)=1$ by using Lemma 2.6 without reference. Therefore $\alpha_{1}-3 \leq 12, \beta_{2}-1 \leq 7, \gamma_{2} \leq 5$. Further the table mentioned in Lemma 2.6 does not give any solution to (12). Thus using the above bounds for $\alpha_{1}, \beta_{2}$ and $\gamma_{2}$ in (12), we get $n \in\{3,6,12,24,30,120,1920\}$ and this is a contradiction as $n>50$ and $\gamma_{1}=0$. Therefore we can assume that $\gamma_{1}=1$ and consequently $\gamma_{2}=0$. Further $\alpha_{1} \geq 2$ since $n>50$. If $\alpha_{1} \in\{2,3\}$, then $n \in\{60,120\}$ and assume that $\alpha_{1} \geq 4$. Thus $\alpha_{2}=\operatorname{ord}_{2}(n+24)=3$ and $n+24=2^{3} \cdot 3^{\beta_{2}}>74$ implying $\beta_{2} \geq 3$. This together with (10) give

$$
3^{\beta_{2}-1}-2^{\alpha_{1}-3} \cdot 5=1 .
$$

We use Lemma 2.6 to get $n=1920$. Thus we have $n \in\{60,120,1920\}$. When $(n, \alpha)=$ $(60,24), N P_{7}\left(g_{n}^{(\alpha)}(x)\right)=\{(0,0),(7,1),(56,9),(60,10)\}$ and when $(n, \alpha)=(1920,24)$, $N P_{2}\left(g_{n}^{(\alpha)}(x)\right)=\{(0,0),(128,127),(384,382),(896,893),(1920,1916)\}$. In both cases the slope of right most edge is $<1$ and by Lemma 2.2 we conclude that $\psi_{n}^{(\alpha)}(x)$ has no linear factor in these cases.

Let $\alpha=25$. Then by (9), if $p \mid n(n+25)$, then $p \in\{2,3,5,13\}$. Further by Lemma 3.2 , we may assume that $5 \nmid n$. Therefore, since $n>50$, we have

$$
\begin{aligned}
n & =2^{\alpha_{1}} \cdot 3, & & \alpha_{1} \geq 5 \\
n+25 & =13^{\delta_{2}}, & & \delta_{2} \geq 2 .
\end{aligned}
$$


By considering above equations modulo 8 , we get $13^{\delta_{2}} \equiv 1$ modulo 8 and hence $\delta_{2}$ is even. Then

$$
\left(13^{\delta_{2} / 2}-5\right)\left(13^{\delta_{2} / 2}+5\right)=2^{\alpha_{1}} \cdot 3
$$

is not possible.

Lemma 3.8. Let $P(n) \geq 3$ and $11 \leq \alpha \leq 50$. Then $\psi_{n}^{(\alpha)}(x)$ with $(n, \alpha) \notin \Omega$ has no linear factor.

Proof. Let $p \mid n$ and $p \geq 3$ and $(n, \alpha) \notin \Omega$. Then $n>50$ by Lemma 3.5. Further by Lemmas 3.2 and 3.7, we may assume that

$$
\nu_{p}(\alpha+1)>\nu_{p}(n) .
$$

Since $\nu_{p}(n) \geq 1$, we have $\nu_{p}(\alpha+1) \geq 2$. This gives $\alpha+1 \in\{18,25,27,36,45,49,50\}$. Also (9) is valid. Let $\alpha_{1}, \beta_{1}, \gamma_{1}$ and $\alpha_{2}, \beta_{2}, \gamma_{2}$ be non-negative integers.

Let $\alpha=17$. Then $P(n(n+17))=3$ by (9). Therefore $n=2^{\alpha_{1}} \cdot 3$ by (13), $P(n) \geq 3$ and $n+17=2^{\alpha_{2}}$. Then $\alpha_{2} \neq 0$ implying $\alpha_{1}=0$ which is a contradiction as $n>50$.

Let $\alpha=24$. By (9), (13) and $P(n) \geq 3$, we have $n=2^{\alpha_{1}} \cdot 5$ and $n+24=2^{\alpha_{2}}$. Since $n>50, \alpha_{1} \geq 4$. Thus $\alpha_{2}=\operatorname{ord}_{2}(n+24)=3$ which is a contradiction since $n>50$.

Let $\alpha=26$. By (99), we have $P(n(n+26)) \leq 5$. This together with (13), $n>50$ and $P(n) \geq 3$ give $n=2^{\alpha_{1}} 3^{\beta_{1}}$ with $\beta_{1} \in\{1,2\}, \alpha_{1} \geq 3$ and $n+26=2^{\alpha_{2}} 5^{\gamma_{2}}$. Since $\alpha_{1} \geq 3$, we have $\alpha_{2}=\operatorname{ord}_{2}(n+26)=1$. This gives

$$
5^{\gamma_{2}}-2^{\left(\alpha_{1}-1\right)} 3^{\beta_{1}}=13 .
$$

Let $\alpha_{1} \geq 4$. Consider the above equation modulus 8 . We have $5 \equiv 5^{\gamma_{2}}(\bmod 8)$ implying $\gamma_{2}$ is odd. On the other hand if we consider modulus 3 , we have $1 \equiv 5^{\gamma_{2}}(\bmod 3)$ implying $\gamma_{2}$ is even. Therefore we can assume that $\alpha_{1}=3$ and this gives $n=72$ since $n>50$. Then $n+26=98$ and hence $P(n(n+26))=7>5$. which is not possible.

Let $\alpha=35$. By (9), we have $P(n(n+35)) \leq 5$. Then by (13) and $P(n) \geq 3$, we have $n=2^{\alpha_{1}} \cdot 3$ and $n+35=2^{\alpha_{2}}$. Then $\alpha_{1}=0$ and $n=3$ which contradicts $n>50$.

Let $\alpha=44$. Then by (9), $P(n) \geq 3$ and by (13), we have $n=2^{\alpha_{1}} \cdot 3$ with $\alpha_{1} \geq 5$ since $n>50$. Further $n+44=2^{\alpha_{2}} 5^{\gamma_{2}} 7^{\delta_{2}}$ with $\alpha_{2}=2$. Then we have

$$
11=5^{\gamma_{2}} 7^{\delta_{2}}-2^{\alpha_{1}-2} \cdot 3
$$

We check the solutions of this equation by Lemma 2.6 and we get $n=96$ since $n>50$. For $(n, \alpha)=(96,44)$, we apply Lemma 2.2 with $p=7$ to conclude that $\psi_{n}^{(\alpha)}(x)$ has no linear factor.

Let $\alpha=48$. Then by (9), (13) and $P(n) \geq 3$, we have $n=2^{\alpha_{1}} \cdot 7$ and $n+48=2^{\alpha_{2}} 5^{\gamma_{2}}$. If $\alpha_{1}=3$, then $n=56, n+48=104$ and $13 \mid(n+48)$ and by (9) , this is not possible. If $\alpha_{1}=4$, then $n=112$ and $(n, \alpha)=(112,48) \in \Omega$. If $\alpha_{1}=5$, then $n=224, n+48=272$ and $17 \mid(n+48)$ and this is not possible by (9). Thus $\alpha_{1} \geq 6$. Then $\alpha_{2}=4$ and we 
have

$$
3=5^{\gamma_{2}}-2^{\alpha_{1}-4} \cdot 7
$$

Taking congruent modulo 4, we conclude that the above equation has no solution.

Let $\alpha=49$. By (9), $P(n) \geq 3, n>50$ and (13), we have $n=2^{\alpha_{1}} \cdot 5$ with $\alpha_{1} \geq 4$. Thus $n+49=3^{\beta_{2}}$ and we have

$$
49=3^{\beta_{2}}-2^{\alpha_{1}} \cdot 5
$$

By considering the above equation modulo 8 , we get $\beta_{2}$ even. Then

$$
\left(3^{\beta_{2} / 2}+7\right)\left(3^{\beta_{2} / 2}-7\right)=2^{\alpha_{1}} \cdot 5 .
$$

This implies $3^{\beta_{2} / 2}-7 \in\{2,4,10,20\}$ which does not give solution to the above equation.

Proof of Theorem 1: Let $11 \leq \alpha \leq 50$ and $\psi_{n}^{(\alpha)}(x)$ has a factor of degree $1 \leq k \leq \frac{n}{2}$. By Lemma 3.4, we may assume that $k=1$. Let $(n, \alpha) \notin \Omega$. By Lemmas 3.5 and 3.8, we may assume that $n>50$ and $P(n)=2$. Then $n=2^{r}>\alpha$. Consider $g_{n}^{(\alpha)}(x)$. The leading coefficient of $g_{n}^{(\alpha)}(x)$ is 1 and its constant term is $(n+\alpha)(n+\alpha-1) \cdots(1+\alpha)$. We apply Lemma 2.8 to see that

$$
\begin{aligned}
\nu_{2}((n+\alpha)(n+\alpha-1) \cdots(1+\alpha)) & =\nu_{2}((n+\alpha) !)-\nu_{2}(\alpha !) \\
& =((n+\alpha)-\sigma(n+\alpha))-(\alpha-\sigma(\alpha))=n-1 .
\end{aligned}
$$

The coefficient of $x^{j}$ in $g_{n}^{(\alpha)}(x)$ is $\left(\begin{array}{c}n \\ j\end{array}\right)(n+\alpha)(n+\alpha-1) \cdots(j+1+\alpha)=\frac{n !}{j !}\left(\begin{array}{c}n+\alpha \\ j+\alpha\end{array}\right)$ and

$$
\begin{aligned}
\nu_{2}\left(\frac{n !}{j !}\left(\begin{array}{l}
n+\alpha \\
j+\alpha
\end{array}\right)\right) \geq \nu_{2}\left(\frac{n !}{j !}\right) & =\nu_{2}(n !)-\nu_{2}(j !)=(n-1)-(j-\sigma(j)) \\
& \geq(n-1)-(j-1)=n-j \text { for } 1 \leq j \leq n-1 .
\end{aligned}
$$

This implies that $N P_{2}\left(g_{n}^{(\alpha)}(x)\right)$ is the edge joining $(0,0)$ and $(n, n-1)$. Therefore $N P_{2}\left(g_{n}^{(\alpha)}(x)\right)=N P_{2}\left(\psi_{n}^{(\alpha)}(x)\right)$ has only one edge with no lattice point. Thus $\psi_{n}^{(\alpha)}(x)$ is irreducible and in particular it has no linear factor. Now we apply Lemma 3.6 to complete the proof of Theorem [1.

\section{Proof of Theorem 3}

The proof depends on the following result which is analogous to a result for $G_{\alpha}(x)=$ $G_{n}^{(\alpha)}(x)$ as proved in [9, Lemma 5.2]. The proof of Lemma 4.1] is exactly the same as [9, Lemma 5.2].

Lemma 4.1. Let $\alpha=u+\frac{1}{2}, 1 \leq k \leq \frac{n}{2}$ and $a_{0}, a_{1}, \ldots, a_{n} \in \mathbb{Z}$. Suppose there is a prime $p$ with

$$
p \mid \prod_{l=0}^{k-1}(1+2 u+2(n-l))(n-l), \quad p \nmid \prod_{l=1}^{k}(1+2 u+2 l)
$$


satisfying

$$
p>\max (2 k, 1+\sqrt{2(u+1)}) \text { and } p \nmid a_{0} a_{n} .
$$

Then $\psi_{n}^{(\alpha)}\left(x^{2}\right)$ does not have a factor of degree in $\{2 k-1,2 k\}$. Further when $n$ is odd and $k=\frac{n-1}{2}, \psi_{n}^{(\alpha)}\left(x^{2}\right)$ does not have a factor of degree $n=2 k+1$.

Proof of Theorem [3: Assume that $\psi_{n}^{(\alpha)}\left(x^{2}\right)$ has a factor of degree $1 \leq l \leq n$. Recall that $\psi_{n}^{(\alpha)}\left(x^{2}\right)$ is a special case of $G_{n}^{(\alpha)}\left(x^{2}\right)$. Let $l \geq 3$. Then by Lemma 2.4, $(u, n) \in\{(1,12),(6,7),(9,113),(10,3),(21,101)\}$ if $l=3$, or $(u, n) \in S$ if $l=4$ or $(u, n)=(44,79)$ if $l=6$. We apply Lemma 4.1 to exclude these possibilities except for $(u, n, l)=(4,9,4)$. Hence we may assume that $l \leq 2$. Then by Lemma 2.5, we have $(u, n) \in T_{0}$.

Let $(u, n)=(38,2)$. We may assume that $\psi_{n}^{(\alpha)}\left(x^{2}\right)=x^{4}+162 a x^{2} \pm 6399$. First, we show that $\psi_{n}^{(\alpha)}\left(x^{2}\right)$ has no linear factor. If not, we get a rational root $r / s$, with $r, s \in \mathbb{Z}, \operatorname{gcd}(r, s)=1, s>0$, of $x^{4}+162 a x^{2} \pm 6399=x^{4}+2 \cdot 9^{2} a x^{2} \pm 9^{2} \cdot 79$. Hence $r^{4}+2 \cdot 9^{2} a r^{2} s^{2} \pm 9^{2} \cdot 79 s^{4}=0$ giving $s=1$ and further $r^{2} \mid 9^{2} \cdot 79$. Also $9^{2} \mid r^{4}$ and hence $r^{2} \in\left\{3^{2}, 9^{2}\right\}$. We have $r^{2} \neq 9^{2}$ else $r^{4}=9^{4} \mid 9^{2} \cdot 79$ which is not possible. Thus $r^{2}=3^{2}$ and we obtain $1+18 a \pm 79=0$ or $18 a \in\{-80,78\}$ which is not possible. Hence $\psi_{n}^{(\alpha)}\left(x^{2}\right)$ has no linear factor. Assume it has an irreducible factor of degree 2. Then, we can write $x^{4}+162 a x^{2} \pm 6399=\left(x^{2}+A_{1} x+A_{0}\right)\left(x^{2}+B_{1} x+B_{0}\right)$ with $A_{0}, B, A_{1}, B_{1} \in \mathbb{Z}$. Then $A_{0} B_{0}= \pm 6399, A_{0} B_{1}+A_{1} B_{0}=0=A_{1}+B_{1}$ giving $B_{1}=-A_{1}$ and hence $A_{0}=B_{0}$ if $A_{1} \neq 0$. If $A_{1} \neq 0$, then $\pm 6399=A_{0} B_{0}=A_{0}^{2}$ which is not possible. Hence $A_{1}=0$ giving $B_{1}=0$ and hence $A_{0}+B_{0}=162 a$ which together with $A_{0} B_{0}= \pm 6399$ gives $\left(A_{0}-B_{0}\right)^{2}=(162 a)^{2} \mp 4 \cdot 6399=18^{2}\left\{(9 a)^{2} \mp 79\right\}$. This imply $\pm 79=(9 a)^{2}-y^{2}=(9 a-y)(9 a+y)$ for some $y>1$. Then $9 a-y= \pm 1$ and $9 a+y= \pm 79$ giving $9 a=( \pm 1 \pm 79) / 2$ which is not possible. Thus $\psi_{n}^{(\alpha)}\left(x^{2}\right)$ is irreducible at $(u, n)=(38,2)$.

Let $(u, n) \in T_{0}-\{(38,2)\}$. For all these pairs $(u, n)$ we apply Lemma 2.2 with suitable primes to conclude that $\psi_{n}^{(\alpha)}\left(x^{2}\right)$ does not have factor in degree 1 . Hence we may assume that $\psi_{n}^{(\alpha)}\left(x^{2}\right)$ has a factor of degree 2 for all $(u, n) \in T_{0}-\{(38,2)\}$. Let $(u, n) \in\left\{(35,2),\left(36,2^{6}\right)\right\}$. We apply Lemma 2.2 with $p=3$ for $(u, n)=(35,2)$ and $p=67$ for $(u, n)=\left(36,2^{6}\right)$ to conclude that $\psi_{n}^{(\alpha)}\left(x^{2}\right)$ does not have factor in degree 2 . For $(u, n)=(11,2)$, we find that vertices of $N P_{3}\left(g_{n}^{(\alpha)}\left(x^{2}\right)\right)$ are given by $\{(0,0),(4,3)\}$ and therefore $N P_{3}\left(g\left(x^{2}\right)\right)$ is same as $N P_{3}\left(\psi_{n}^{(\alpha)}\left(x^{2}\right)\right)$. Hence by Lemma 2.1, $\psi_{n}^{(\alpha)}\left(x^{2}\right)$ does not have factor of degree 2 when $(u, n)=(11,2)$. For all other pairs $(u, n)$, we can always find integers $a_{0}, a_{1}, \ldots, a_{n}$ with $\left|a_{0}\right|=\left|a_{n}\right|=1$ such that $\psi_{n}^{(\alpha)}\left(x^{2}\right)$ has a quadratic factor except for $(u, n)=\left(44,2^{12}\right)$ (see Table 2) by the method described in Lemma 3.6.

Proof of Corollary 1.1: Let $\alpha=u+\frac{1}{2}$ where $u$ is an integer. Suppose $\psi_{n}^{(\alpha)}(x)$ has a factor of degree $k$. Then $\psi_{n}^{(\alpha)}\left(x^{2}\right)$ has a factor of degree $2 k$. Therefore by Theorem 3 , we have $(u, n) \in \Omega_{1}, k=1$ and the assertion follows from Theorem 3 immediately. 


\section{Galois Groups: Proof of Theorem 2}

We will use a result of Hajir [5] which gives a criterion for an irreducible polynomial to have large Galois group using Newton polygons. We restate the result which is [5, Lemma 3.1].

Lemma 5.1. Let $f(x)=\sum_{j=0}^{m}\left(\begin{array}{c}m \\ j\end{array}\right) c_{j} x^{j} \in \mathbb{Q}[X]$ be an irreducible polynomial of degree $m$. Let $p$ be a prime with $\frac{m}{2}<p<m-2$ such that

(i) $\operatorname{ord}_{p}\left(c_{j}\right) \geq 0$ for $j=0,1, \ldots, m$,

(ii) $\operatorname{ord}_{p}\left(c_{0}\right)=1$

(iii) $\operatorname{ord}_{p}\left(c_{j}\right) \geq 1$ for $0 \leq j \leq m-p$,

(iv) $\operatorname{ord}_{p}\left(c_{p}\right)=0$.

Then the Galois group of $f$ contains $A_{m}$. Further Galois group is $A_{m}$ if $\operatorname{disc}(f) \in \mathbb{Q}^{* 2}$ and $S_{m}$ otherwise.

We shall always assume that $\alpha=u+\frac{1}{2}$ in this section where $u$ is an integer $\geq 1$. We define

$$
\mathcal{L}_{n}^{(u)}(x)=\sum_{j=0}^{n}\left(\begin{array}{l}
n \\
j
\end{array}\right)(1+2(u+n))(1+2(u+n-1)) \cdots(1+2(u+j+1)) x^{j} .
$$

We observe that $\mathcal{L}_{n}^{(u)}(2 x)=2^{n} n ! L_{n}^{(\alpha)}(-x)$ and thus the Galois group of $\mathcal{L}_{n}^{(u)}(x)$ and $L_{n}^{(\alpha)}(x)$ are same.

We shall be applying the above lemma with $f(x)=\mathcal{L}_{n}^{(u)}(x)$. In [15], Schur showed that the discriminant of $\mathcal{L}_{n}^{(u)}(x)$ is given by

$$
D_{n}^{(u)}:=\operatorname{Disc}\left(\mathcal{L}_{n}^{(u)}(x)\right)=\prod_{j=2}^{n} j^{j}\left(\frac{2 u+1}{2}+j\right)^{j-1} .
$$

We write $D_{n}^{(u)}=b Y^{2}, Y \in \mathbb{Q}$ with

$$
b= \begin{cases}\frac{3 \cdot 5 \cdots n \cdot(2 u+1+4)(2 u+1+8) \cdots(2 u+1+2(n-1))}{2^{\delta}} & \text { if } n \equiv 1,3(\bmod 4) \\ \frac{3 \cdot 5 \cdots(n-1) \cdot(2 u+1+4)(2 u+1+8) \cdots(2 u+1+2 n)}{2^{\delta}} & \text { if } n \equiv 0,2(\bmod 4)\end{cases}
$$

where $\delta=0$ if $n \equiv 0,1(\bmod 4)$ and 1 if $n \equiv 2,3(\bmod 4)$. Observe that $b$ is never a square when $n \equiv 2,3(\bmod 4)$. In the next lemma, we find all pairs $(u, n)$ such that $b$ is a square.

Lemma 5.2. Let $u \leq \max \left(45, \frac{4 n}{3}\right)$. The pairs $(u, n)$ for which $b$ given by (14) is a square are $(u, n)=(u, 1)$ where $1 \leq u \leq 45$ in which cases $b=1$.

Proof. We may assume that $n \equiv 0,1(\bmod 4)$. Let $\eta=0,1$ according as $n \equiv 0,1$ modulo 4 , respectively. Let $x=\frac{1+2(u+n-\eta)}{1.048}$ and $x \geq 887$. Then $n>198$ since $u \leq \max \left(45, \frac{4 n}{3}\right)$. Further $x>\max (n-1+\eta, 2 u+4)$. Therefore by Lemma 2.9, the interval $(\max (n-1+\eta, 2 u+4), 1+2(u+n-\eta)]$ contains a prime $p$ congruent to 
$1+2 u$ modulo 4. Further $2 p>1+2(u+n-\eta)$ since $p>x$. Hence $b$ is not a square. Therefore we may now suppose that $x=(1+2(u+n-\eta)) / 1.048<887$ or $1+2(u+n-\eta) \leq 929$.

We have $8(u+n) / 7 \geq 2 u$ if $u \leq 4 n / 3$. Hence taking $m=1+2(u+n-\eta)$, we get that $4 m / 7+5 \geq 2 u+5-4 / 7>2 u+4$ if $u \leq 4 n / 3$. Also $4 m / 7+5>n$. For $m \geq 158$, we also have $4 m / 7 \geq 2 \cdot 45$. Hence for $158 \leq m \leq 929$, we check that the interval $(4 \mathrm{~m} / 7+5, m]$ contain both primes congruent to 1 and 3 modulo 4 . Thus we may suppose that $m=1+2(u+n-\eta) \leq 157$.

Let $2 u+4 \leq n$. Then $\max (2 u+4, n)=n<m / 2$. For $7 \leq m \leq 157$ and $m$ odd, we check that the interval $(\mathrm{m} / 2, \mathrm{~m}]$ contain both primes congruent to 1 and 3 modulo 4 except for $m=11$. Hence for $1+2(u+n-\eta) \leq 157, b$ is not a square except when $1+2(u+n-\eta)=11$ or $1+2(u+n) \leq 6$. These cases can be excluded since $n \geq 2 u+4$.

Let $n \leq 2 u+3$. Then $3 n-2-2 \eta \leq 1+2(u+n-\eta) \leq 157$. Thus $n \leq 53$. We check that for primes $\leq 157$, gaps between consecutive primes in the same residue modulo 4 is at most 24 . Hence from (14), we obtain that $b$ is not square if $2(n-1) \geq 24$ or $n \geq 13$. Thus we may suppose that $n \leq 12$. Then $n \in\{1,4,5,8,9,12\}$. For these values of $n$, we have $u \leq \max (45,4 n / 3)=45$ and we check that $b$ is not a square unless $(u, n)=(u, 1)$ where $1 \leq u \leq 45$. Hence the assertion.

Lemma 5.3. Let $n>1, u \leq \max \left(45, \frac{4 n}{3}\right)$ and $\mathcal{L}_{n}^{(u)}(x)$ be an irreducible polynomial. Suppose there exists a prime $p$ with $\frac{n}{2}<p<n-2$ such that

$$
p \| \prod_{l=n-p+1}^{p}(1+2(u+l)),
$$

then the Galois group of $\mathcal{L}_{n}^{(u)}(x)$ is $S_{n}$.

Proof. We apply Lemma 5.1 with $f(x)=\mathcal{L}_{n}^{(u)}(x)$. Then

$$
c_{j}=(1+2(u+n))(1+2(u+n-1)) \cdots(1+2(u+j+1)) .
$$

Since $n / 2<p<n-2$, there are at most 2 terms in

$$
1+2(u+1), \ldots, 1+2(u+n)
$$

divisible by $p$. By (15) and $2 p-n<p$, there is exactly one $l_{p}$ with $n-p+1 \leq l_{p} \leq p$ and $p \|\left(1+2\left(u+l_{p}\right)\right)$. This together with $l_{p}-p \leq 0$ and $l_{p}+p>n$ implies $1+2\left(u+l_{p}\right)$ is the only term exactly divisible by $p$ in $1+2(u+1), \ldots, 1+2(u+n)$. Hence $p \nmid c_{p}$ since $l_{p} \leq p$. Further for $0 \leq j \leq n-p$, we have $\operatorname{ord}_{p}\left(c_{j}\right)=\operatorname{ord}_{p}\left(1+2\left(u+l_{p}\right)\right)=1$. Hence all the assumptions in Lemma 5.1 are satisfied. Finally we apply Lemmas 5.1, 5.2 and $n>1$ to get the assertion of Lemma 5.3 .

Lemma 5.4. Let $u \leq \max \left(45, \frac{4 n}{3}\right)$ and $\alpha=u+\frac{1}{2}$. Suppose $\mathcal{L}_{n}^{(u)}(x)$ be irreducible. Then the Galois group of $\mathcal{L}_{n}^{(u)}(x)$ is $S_{n}$. 
Proof. Let $n \leq 130$ and $u \leq \max \left(45, \frac{4 n}{3}\right)$. We apply Lemma 5.3 for these pairs of $(u, n)$. We check that all these pairs with $n \geq 40$ are excluded. Out of the remaining we are left with 619 pairs of $(u, n)$ for which Lemma 5.3 is not satisfied. For these 619 pairs, we compute Galois group directly using MAGMA. Hence we may now suppose that $n>130$. Since $1+2(u+n)<(n / 2)^{2}$, by Lemma 5.3, it suffices to choose a prime $p \in(n / 2, n-2)$ with $p \mid\left(1+2\left(u+l_{p}\right)\right)$ for some $l_{p}$ such that $n-p+1 \leq l_{p} \leq p$. For each $p \in(n / 2, n-2)$, we write

$$
1+2(u+n)=t_{p} p+r_{p}, 0 \leq r_{p}<p .
$$

It suffices to find a prime $p \in(n / 2, n-2)$ such that $p$ divides one of $r_{p}+2, r_{p}+$ $4, \ldots, r_{p}+2(2 p-n)$. We now restrict to $p \in(2 n / 3, n-2)$.

Suppose $r_{p}$ is odd for some $p \in(2 n / 3, n-2)$. Then $r_{p}+2(2 p-n) \geq p$ if $r_{p} \geq$ $2 n-3 p$ which is true since $2 n-3 p<0 \leq r_{p}$. This, together with $r_{p}<p$ and $r_{p}$ odd implies $p=r_{p}+2 i$ some $i \leq 2 p-n$. Hence we may now assume that $r_{p}$ is even for each $p \in(2 n / 3, n-2)$. Write $r_{p}=2 r_{p}^{\prime}$ with $0 \leq r_{p}^{\prime} \leq(p-1) / 2$. Then $r_{p}+2, r_{p}+4, \ldots, r_{p}+2(2 p-n)$ is given by $2\left(r_{p}^{\prime}+1\right), 2\left(r_{p}^{\prime}+2\right), \ldots, 2\left(r_{p}^{\prime}+2 p-n\right)$. If $r_{p}^{\prime}+2 p-n \geq p$ for some $p \in(2 n / 3, n-2)$, then we are done. Hence assume that $r_{p}^{\prime}+2 p-n<p$ implying $r_{p}=2 r_{p}^{\prime} \leq 2 n-2 p-2$ for each $p \in(2 n / 3, n-2)$. Further

$$
1+2(u+n)<\left(t_{p}-2\right) p+2 n \text { and } t_{p} \text { is odd for each } p \in(2 n / 3, n-2)
$$

since $1+2(u+n)$ is odd.

We now write $P_{1}, P_{2}$ for the least prime and maximum prime in $(2 n / 3, n-2)$, respectively. Then $P_{1}=p_{\pi([2 n / 3])+1}>2 n / 3$ and $P_{2}=p_{\pi(n-3)} \leq n-3$. We first show the following:

$$
3 P_{2}-P_{1}>2 n
$$

For $130<n \leq 1000$, we check that the above assertion holds. Assume $n>1000$. By [6], there is a prime in $(m, 1.05 m)$ for every $m \geq 213$. Taking $m=\lfloor 2 n / 3\rfloor$, we get $P_{1}<1.05 \cdot 2 n / 3=0.7 n$. Again taking $m=\left\lfloor\frac{n-3}{1.05}\right\rfloor=\lfloor 20(n-3) / 21\rfloor$, we get $P_{2} \geq 20(n-3) / 21$. Thus $3 P_{2}-P_{1} \geq 20(n-3) / 7-0.7 n>2 n$.

Case I: Let $u$ be such that $1+2(u+n)<3 P_{2}$. Taking $p=P_{2}$, we get $1+2(u+n)=$ $t_{p} p+r_{p}<3 p$ giving $t_{p} \in\{0,1,2\}$ implying $t_{p}=1$ since $t_{p}$ is odd. Hence $r_{p}=$ $1+2(u+n)-p>2 n-p>2 n-2 p$ which is a contradiction. Thus $1+2(u+n) \geq 3 P_{2}$.

Case II: Let $u$ be such that $3 P_{2} \leq 1+2(u+n)<5 P_{1}$. Taking $p=P_{1}$, we get $3 p<3 P_{2} \leq 1+2(u+n)=t_{p} p+r_{p}<5 p$ giving $t_{p}=3$ since $t_{p}$ is odd and $r_{p}<p$. This gives $3 P_{2} \leq(3-2) P_{1}+2 n$ or $3 P_{2}-P_{1} \leq 2 n$ which contradicts (17). Thus $1+2(u+n) \geq 5 P_{1}$.

Case III: Let $u$ be such that $5 P_{1} \leq 1+2(u+n)<5 P_{2}$. Observe that $3 P_{2}<5 P_{1}$ since $P_{1}>2 n / 3$ and $P_{2}<n-2$. Taking $p=P_{2}$, we get $3 p<5 P_{1} \leq 1+2(u+n)=$ $t_{p} p+r_{p}<5 p$ giving $t_{p}=3$ since $t_{p}$ is odd and $r_{p}<p$. Further $5 P_{1} \leq(3-2) P_{2}+2 n$. This is a contradiction since $10 n / 3<5 P_{1} \leq P_{2}+2 n<3 n$. Thus $1+2(u+n) \geq 5 P_{2}$. 
Case IV: Let $u$ be such that $5 P_{2} \leq 1+2(u+n)<7 P_{1}$. Taking $p=P_{1}$, we get $5 p<5 P_{2} \leq 1+2(u+n)=t_{p} p+r_{p}<7 p$ giving $t_{p}=5$. This gives $5 P_{2} \leq(5-2) P_{1}+2 n$ i.e $3 P_{2}-P_{1}+2\left(P_{2}-P_{1}\right) \leq 2 n$ which contradicts (17).

Thus $1+2(u+n) \geq 7 P_{1}$. Since $P_{1}>2 n / 3$, we have $P_{1} \geq 2 n / 3+1 / 3$ giving $1+2(u+n) \geq 7(2 n / 3+1 / 3)$ implying $u>4 n / 3$. Since $u \leq \max (45,4 n / 3)$ and $n \geq 130$, this is not possible. Hence the assertion.

Proof of Theorem 2; By [9, Corollary 1.1], we see that $\mathcal{L}_{n}^{(u)}(x)$ is irreducible except for $(u, n)=(10,3)$. For $(u, n)=(10,3)$, we check that the Galois group is $\mathbb{Z}_{2}$. For $(u, n) \neq(10,3)$, the assertion now follows from Lemma 5.4.

\section{REFERENCES}

[1] J. Cullinan and F. Hajir, Primes of prescribed congruence class in short intervals, Integers 12(2012), Paper No. A56, 4 pp.

[2] M. G. Dumas, Sur quelques cas d'irréductibilité des polynomes à coefficients rationnels, J. Math. Pures Appl., 2 (1906), 191-258.

[3] M. Filaseta, The irreducibility of all but finitely many Bessel polynomials, Acta Math., 174 (1995), 383-397.

[4] M. Filaseta, C. Finch and J. R. Leidy, T. N. Shorey's influence in the theory of irreducible polynomials, Diophantine Equations, Narosa Publ., New Delhi (2008), 77-102.

[5] F. Hajir, On the Galois group of generalized Laguerre polynomials, J. Théor. Nombres Bordeaux, 17(2) (2005), 517-525.

[6] H. Harborth and A. Kemnitz, Calculations for Bertrand's Postulate, Mathematics Magazine, 54(1981), No. 1, 33-34.

[7] H. Hasse, Number theory, Classics in Mathematics, Springer-Verlag, Berlin 2002.

[8] S. Laishram and T. N. Shorey, Extensions of some irreducibility results, Indag. Math., 21 (2011), 87-105.

[9] S. Laishram, S. G Nair and T. N. Shorey, Irreducibility of Generalized Laguerre Polynomials $L_{n}^{\left(\frac{1}{2}+u\right)}(x)$ with integer $u$, J. Number Theory, 160 (2016), 76-107.

[10] S. Laishram, On the Galois groups of generalized Laguerre Polynomials, Hardy Ramanujan Journal, 37 (2015), 8-12.

[11] D. H. Lehmer, On a problem of Störmer, Illinois J. Math. 8 (1964), 57-79.

[12] F. Luca and F. Najman, On the largest prime factor of $x^{2}-1$, Math. Comp. 80 (2011), 429-435.

[13] F. Luca and F. Najman, Errata to On the largest prime factor of $x^{2}-1$, Math. Comp. 83 (2014), 337.

[14] I. Schur, Einige Sätze über Primzahlen mit Anwendungen auf Irreduzibilitätsfragen, II, Sitzungsber. Preuss. Akad. Wiss. Berlin Phys.-Math. Kl. 14 (1929), 370-391.

[15] I. Schur, Affektlose Gleichungen in der Theorie der Laguerreschen und Hermitschen Polynome, J. Reine Angew. Math. 165 (1931), 52-58.

[16] G. Szego, Orthogonal Polynomials, Amer. Math. Soc. Colloq. Publ. Series Vol 23, Amer. M. Soc, Providence RI, 1975, Fourth Edition.

[17] B.M.M de Weger, Solving exponential Diophantine equations using lattice basis reduction algorithms, J. Number Theory 26 (1987), 325-367. 
Stat-Math Unit, India Statistical Institute, 7, S. J. S. Sansanwal Marg, New Delhi, 110016, INDIA

E-mail address: shanta@isid.ac.in

Department of Mathematics, IIT Bombay, Powai, Mumbai 400076, India

E-mail address: saranya@math.iitb.ac.in

E-mail address: shorey@math.iitb.ac.in 